\begin{tabular}{|c|l|}
\hline Title & Grain boundary engineering of austenitic steel PNC316 for use in nuclear reactors \\
\hline Author(s) & Sekine, M.; Sakaguchi, N.; Endo, M.; Kinoshita, H.; W atanabe, S.; Kokawa, H.; Y amashita, S.; Y ano, Y.; Kawai, M. \\
\hline Citation & $\begin{array}{l}\text { Journal of Nuclear Materials, 414(2), 232-236 } \\
\text { https:/doi.org/10.1016/.jnucmat.2011.03.049 }\end{array}$ \\
\hline Issue Date & 2011-07-15 \\
\hline Doc URL & http://hdl.handle.net/2115/47606 \\
\hline Type & article (author version) \\
\hline File Information & JNM414_2_232-236.pdf \\
\hline
\end{tabular}

Instructions for use 


\section{Grain Boundary Engineering of Austenitic Steel PNC316 for use in Nuclear}

\section{Reactors}

M.Sekine ${ }^{1}$, N.Sakaguchi*1, M.Endo ${ }^{1}$, H.Kinoshita ${ }^{1}$, S.Watanabe ${ }^{1}$, H.Kokawa ${ }^{2}$, S.Yamashita ${ }^{3}$, Y.Yano $^{3}$, M.Kawai ${ }^{4}$

${ }^{1}$ Center for Advanced Research of Energy and Materials, Faculty of Engineering, Hokkaido University, Kita-13, Nishi-8, Kita-ku, Sapporo, Hokkaido, 060-8628, Japan

${ }^{2}$ Graduate School of Engineering, Tohoku University, Aramaki-aza-Aoba, Aoba-ku, Sendai 980-8579, Japan

${ }^{3}$ Materials Monitoring Section, Fuels and Materials Department, O-arai Research and Development Center, Japan Atomic Energy Agency (JAEA), 4002 Narita-cho, Oarai-machi, Ibaraki 311-1393, Japan

${ }^{4}$ Institute of Materials Structure Science, High Energy Accelerator Research Organization, Oho, Tsukuba-shi, Ibaraki-ken 305-0801, Japan

* Corresponding Author TEL: +81-011-706-6768, FAX: +81-011-706-6768

E-mail: sakagu@ufml.caret.hokudai.ac.jp, (N. Sakaguchi)

Present address: CAREM, Faculty of Engineering, Hokkaido University, Kita-13, Nishi-8, Kita-ku, Sapporo, Hokkaido, 060-8628, Japan 


\section{Abstract}

Austenitic stainless steel PNC316 was subjected to grain boundary engineering (GBE). It was found that the grain boundary engineered PNC316 (PNC316-GBEM) had a coincidence site lattice (CSL) fraction of $86 \%$ and that the network of random grain boundaries was perfectly divided by the CSL boundaries. The thermal stability and the void swelling behavior of PNC316-GBEM were investigated by means of SEM and TEM analyses. After thermal aging at $973 \mathrm{~K}$ for $100 \mathrm{~h}$, structural changes were observed neither in the grain boundary networks of PNC316-GBEM nor in another sample of PNC316-GBEM subjected to $20 \%$ additional cold rolling, PNC316-GBEM20\%CW. PNC316-GBEM showed a higher void swelling rate than as-received PNC316 (PNC316-AS). However, with additional 20\% cold rolling after GBE, the void swelling rate decreased to as low as that of PNC316-AS.

KEYWORDS: grain boundary engineering, coincidence lattice site boundary, random grain boundary, cold working, electron irradiation, PNC316 


\section{Introduction}

Second- and third-generation nuclear reactors are now in operation in most nuclear power plants. In the near future, the fourth-generation (Gen IV) nuclear reactors will be required to ensure improved nuclear safety, proliferation resistance, etc. [1]. Supercritical water (SCW) has attracted much attention because SCW boiler power plants were implemented to increase the efficiency of fossil-based power plants. The SCW reactor (SCWR) design has been selected as one of the Gen IV reactor concepts because SCWRs have higher thermal efficiency and simpler plant design than the current light water reactors (LWRs). Reactor operating conditions are expected to be as follows: a core coolant temperature between $553 \mathrm{~K}$ and $893 \mathrm{~K}$ at a pressure of $25 \mathrm{MPa}$ and maximum neutron damage levels to any replaceable or permanent core component of $15 \mathrm{dpa}$ (thermal reactor design) and $100 \mathrm{dpa}$ (fast reactor design) [2]. The main problems of core component materials at high temperatures are associated with void swelling, high-temperature strength and creep strength. On the other hand, austenitic stainless steels are susceptible to irradiation-assisted stress corrosion cracking (IASCC) $[3,4]$ at low temperatures. It is well known that cracks formed by IASCC propagate mainly along the grain boundaries.

Many grain boundary studies have revealed that grain boundary phenomena such as grain boundary diffusion [5], sliding [6], precipitation [7-9], and corrosion [10, 11] depend strongly on the grain boundary structure and character [12]. It is generally accepted that some low-energy grain 
boundaries such as coincidence site lattice (CSL) boundaries, unlike high-energy random grain boundary boundaries, are highly resistant to grain boundary deterioration. The concept of grain boundary design and control [13] has been developed and refined as grain boundary engineering (GBE) [14-19]. The GBE method is one of the thermo-mechanical treatments that can significantly improve material properties without changing the alloy composition or adding minor elements. It has been reported in the literature that the random grain boundary network in austenitic stainless steel is completely disrupted after GBE processing when the CSL ratio exceeds $80 \%$ [20]. In these materials, the GBE method improves the creep resistance [19] and the grain boundary corrosion properties [20].

In the present study, we applied a GBE technique to develop a high-performance material for nuclear reactor components. PNC316, a modified type-316 austenitic stainless steel developed for the prototype fast breeder reactor (FBR) MONJU, was used as the base material. The thermal stability of the grain boundary network and the microstructure of the grain boundary engineered (GBEed) PNC316 were investigated by means of SEM and TEM analyses. We also evaluated the effect of additional cold rolling on the grain boundary networks in the GBEed PNC316. The void swelling behavior was investigated by means of electron irradiation using a high-voltage electron microscope (HVEM).

\section{Experimental procedure}


The composition of PNC316 used in the present study is listed in Table 1. The procedure

of GBE for PNC316 was as follows. As-received PNC316 (PNC316-AS), which had been subjected to $20 \%$ cold working, was solution-annealed at $1373 \mathrm{~K}$ for $0.5 \mathrm{~h}$. The sample was then subjected to $3 \%$ cold rolling and was finally annealed again at $1320 \mathrm{~K}$ for $3 \mathrm{~h}$ to enhance the fraction of the CSL boundaries to over 80\% (PNC316-GBEM). Some pieces of the PNC316-GBEM sample were subjected again to $20 \%$ cold rolling to develop a highly dense dislocation network in the matrix (PNC316-GBEM20\%CW). Electron backscatter diffraction (EBSD) patterns were acquired from each specimen by an SEM-EBSD instrument. To confirm the stability of the microstructure and grain boundary network, a thermal aging test was carried out. We chose a temperature of $973 \mathrm{~K}$ as the aging temperature, which was slightly higher than the operating temperature of the FBR and SCWR. The period of the aging test was set to be $100 \mathrm{~h}$, which was long enough to allow us to investigate the stability of the microstructure. The microstructural changes in the specimens were determined by a $200 \mathrm{kV}$ TEM. To examine the void swelling behavior in each sample, the electron irradiation was carried out using the 1.25 MV multi-beam HVEM (MB-HVEM) installed at Hokkaido University. The irradiation was performed at $823 \mathrm{~K}$ and up to $6 \mathrm{dpa}$ with a damage rate of $1 \times 10^{-3} \mathrm{dpa} / \mathrm{s}$. Kenfield et al. indicated that there is a good correlation between the electron and neutron irradiation void swelling results at temperatures above 723K [21]. Makin et al. also confirmed that both of these swelling rates agreed within a factor of two over the temperature range 
723-843K without any temperature shifts or correction factors [22]. The present experimental

condition was adequate to simulate the void swelling behavior in a nuclear reactor.

\section{Results}

\section{3-1 Grain boundary character distribution (GBCD)}

The grain boundary character distribution (GBCD) obtained by SEM-EBSD analysis of PNC316-AS, PNC316-GBEM and PNC316-GBEM20\%CW are presented in Fig. 1 (a-c). In the figures, the black and gray lines indicate random and CSL boundaries $(\Sigma \leq 29)$, respectively. The CSL fractions of PNC316-AS, PNC316-GBEM, and PNC316-GBEM20\%CW were evaluated as $24 \%, 86 \%$ and $41 \%$ (apparently by EBSD), respectively. We found that PNC316-GBEM has the highest CSL fraction (86\%) and that the network of random grain boundaries was perfectly divided by the CSL boundaries. The EBSD result showed that by subjecting PNC316-GBEM to additional $20 \%$ cold rolling the CSL fraction apparently decreased to $41 \%$ because of the limitation of spatial resolution that cannot distinguish between the intrinsic misorientation of a grain boundary from an extrinsic one caused by rolling-introduced dislocations on or close to the grain boundary; however, the measured value of the CSL fraction was twice that found in PNC316-AS. After thermal aging at $973 \mathrm{~K}$ for $100 \mathrm{~h}$, we could not find any structural changes in the grain boundary networks as shown in Fig. 1 (d-f). The CSL fractions of thermal-aged PNC316-AS, PNC316-GBEM, and PNC316-GBEM20\%CW were $25 \%, 86 \%$, and $45 \%$, respectively. The grain boundary network 
introduced by GBE was quite stable without any recrystallization or grain coarsening during the thermal aging.

\section{3-2. Microstructural observation before and after thermal aging}

The microstructures of PNC316-GBEM and PNC316-GBEM20\%CW are shown in Fig. 2

(a, b). After GBE at high temperature, few dislocations were observed in the grains. We could not find any carbide in the grains or on the grain boundaries. After the additional $20 \%$ cold rolling, a high frequency of dislocation networks was introduced, and many deformation twins were also seen. Some deformation twins intersected the grain boundary, as shown in the figure. Fig. 2 (c, d) shows the microstructures of PNC316-GBEM and PNC316-GBEM20\%CW after thermal aging at $973 \mathrm{~K}$ for $100 \mathrm{~h}$. PNC316-GBEM subjected to aging showed continuous carbide precipitates along the grain boundaries, and some small carbides were seen on the dislocations. In the PNC316-GBEM20\%CW, highly dense dislocation networks remained without any recovery or recrystallization after the aging. Coarse carbide precipitates were seen along the grain boundaries, and the formation of many fine carbides was clearly indicated on the dislocations as shown in the magnified image of the grain. As expected, the dislocations were pinned against the fine carbides; therefore, no recovery of the dislocations occurred.

\section{3-3. Void swelling behavior under electron irradiation}

Figure 3 shows the TEM images of (a) PNC316-AS, (b) PNC316-GBEM and (c) 
PNC316-GBEM20\%CW after the electron irradiation at $823 \mathrm{~K}$ to $6 \mathrm{dpa}$. Many small voids were observed in all of the specimens after the electron irradiation. The mean void size in PNC316-AS was smaller than that in PNC316-GBEM, whereas the number density of the void was slightly higher in PNC316-AS. When PNC316-GBEM was subjected to the additional $20 \%$ cold rolling process, the mean diameter of the voids decreased to a size similar to that found in PNC316-AS.

The dose dependence of the void swelling in each specimen is plotted in Fig. 4. It was shown that PNC316-GBEM had a higher void swelling rate than the other two specimens; thus, the amount of void swelling was highest after the electron irradiation to $6 \mathrm{dpa}$. By applying an additional $20 \%$ cold rolling after GBE, the void swelling rate was improved, reaching low levels similar to those of PNC316-AS. The amount of void swelling was lowest in PNC316-GBEM20\%CW after the electron irradiation to 6 dpa.

\section{Discussion}

\section{4-1. Effect of cold working on CSL fraction}

The CSL fraction of austenitic stainless steels PNC316 increased from $24 \%$ to $86 \%$ after thermo-mechanical GBE. This procedure introduced a discontinuous network of random grain boundaries. By applying an additional 20\% cold rolling to PNC316-GBEM, the CSL fraction apparently decreased to $41 \%$, although the absolute values of the CSL frequency were twice that for PNC316-AS. A comparison of the GBCDs of the PNC316-GBEM and PNC316-GBEM20\%CW 
(Fig. 1 (b) and (c)) revealed that the grain size distribution and the total area of grain boundary remained almost unchanged. This means that grain growth and/or recrystallization did not occur and that the highly dense dislocations remained in the grains and on the grain boundaries. Some of the dislocations introduced by the additional cold rolling could be absorbed into the grain boundaries so as to change the grain boundary structures and misorientations, and some of the dislocations may stay on and/or close to the grain boundaries. EBSD cannot distinguish between the intrinsic misorientation of a grain boundary from the extrinsic one caused by rolling-introduced dislocations on or close to the grain boundary. In the present EBSD analysis, a grain boundary is regarded as a random one, if the sum of the measured apparent intrinsic and extrinsic misorientations exceeds the deviation angle ( $\Delta \theta_{\operatorname{Max}}$ ) from an exact CSL relationship with a value of $\Sigma$. According to Brandon's criterion [23], the maximum deviation angle of the CSL boundaries is determined by

$$
\Delta \theta_{\text {Max }}=\frac{15^{\circ}}{\sqrt{\Sigma}} .
$$

It is reported that CSL boundaries resist the change in the intrinsic misorientations to maintain their as-GBEed low-energy structures during deformation because of the much lower absorption rates of lattice dislocations than random boundaries [24, 25]. Therefore, the as-GBEed GBCD could have remained in PNC316-GBEM20\%CW in spite of the apparent reduction in the CSL fraction, which is a result of the limitation of the spatial resolution of EBSD. Additionally, deformation twins during the cold working may have some beneficial effects on the disconnection of random boundary 
networks. It was seen that some grain boundaries intersected with the deformation twins as shown in

Fig. 2 (b). For example, a $\Sigma 45$ CSL boundary, which is regarded as a random one with $\Sigma \leq 29$ in the present study could be partially changed to a $\Sigma 15$ CSL boundary after the intersection; this result indicates that a local conversion from a random boundary to a CSL boundary occurs, resulting in subdivision of a random boundary. For the above reasons, it is speculated that PNC316-GBEM20\%CW could maintain the as-GBEed GBCD with a discontinuous random boundary network that is divided by the CSL boundaries. Therefore, it is expected that PNC316-GBEM20\%CW will have better grain boundary corrosion properties under irradiation than PNC316-AS, although the CSL fraction decreased apparently to $41 \%$ after the $20 \%$ cold working. As shown in Fig.2 (b) and (d), the dislocation cell structure was introduced by $20 \%$ cold working after GBE treatment and some cell walls intersected the grain boundary. These dislocation cells lead to a local misorientation due to a slight rotation across the cell walls. Nevertheless, no structural change from the CSL to the random grain boundary as indicated by the EBSD was seen by TEM; only some steps were formed at the boundaries. This result corresponds to the above expectation. However, the area observed by the TEM in the grain boundary network was extremely limited, as shown in Fig. 1. Further TEM analysis for the specific grain boundaries in GBCD will be required to confirm the above result. 


\section{4-2. Effect of cold working on void swelling}

As mentioned above, the void swelling rate increased after the GBE treatment on the austenitic stainless steels PNC316. There is a noticeable difference between the densities of dislocations in the grains of PNC316-AS and PNC316-GBEM. The dislocation density in PNC316-GBEM was much lower than that of PNC316-AS because of the solution annealing prior to GBE. In the case of the modified SUS316, it has been clarified that its void swelling behavior was improved, with increases in the degree of cold working up to 50\% due to the formation of the highly dense dislocation network [26]. It was suggested that the supersaturated concentration of vacancy decreased when the dislocation density increased because the highly dense dislocations acted as sink sites for the point defects generated by the irradiation. The compositions of both modified SUS316 and PNC316 were almost similar; therefore, the void swelling resistance of PNC316-GBEM (with low dislocation density) was degraded, compared with PNC316-AS (with high dislocation density). We also applied the additional $20 \%$ cold rolling to PNC316-GBEM to improve the swelling behavior due to the introduction of a highly dense dislocation network. As a result, the void swelling rate decreased to the same level at which it was measured prior to GBE. It was also found that the amount of the void swelling in PNC316-GBEM20\%CW was slightly lower than that in PNC316-AS although both steels were subjected to $20 \%$ cold working. Cold drawing was applied to PNC316-AS whereas cold rolling was applied to PNC316-GBEM20\%CW. It would be expected that the density 
or structure of the dislocation network might be slightly different; however, it was difficult to

distinguish these two dislocation structures by TEM.

\section{Conclusion}

Austenitic stainless steel PNC316 was subjected to GBE. PNC316-GBEM had the highest CSL fraction (86\%), and the network of random grain boundaries was perfectly divided by the CSL boundaries. When an additional 20\% cold rolling was applied to PNC316-GBEM, the CSL fraction apparently decreased to $41 \%$ because of the limitation of the spatial resolution of the EBSD that cannot distinguish an intrinsic misorientation of a grain boundary from an extrinsic one caused by rolling-introduced dislocations. After thermal aging at $973 \mathrm{~K}$ for $100 \mathrm{~h}$, it was revealed that the grain boundary network introduced by GBE was stable, without any recrystallization or grain coarsening. It was indicated that the void swelling rate increased after GBE of PNC316 because of the annihilation of the highly dense dislocation network during the solution annealing prior to the GBE. After 20\% cold rolling PNC316-GBEM, the void swelling rate decreased again to levels similar to those for PNC316-AS. We conclude that a discontinuous random grain boundary network can be introduced in the austenitic stainless steel PNC316 by means of GBE, without reducing its high resistance to void swelling. 


\section{Acknowledgements}

The authors gratefully thank Prof. H. Takahashi for his insights. The authors also acknowledge Mr.

K. Sugawara and Mr. K. Ohkubo for their technical support in the HVTEM operations.

\section{References}

[1] Y. Oka, S. Koshizuka, J. Nucl. Sci. Technol. 38 (2001) 1081-1089.

[2] G.S. Was, P. Ampronrat, G. Gupta, S. Teysseyre, E.A. West, T.R. Allen, K. Sridharan, L. Tan, Y.

Chen, X. Ren, C. Pister, J. Nucl. Mater. 371 (2007) 176-201.

[3] Z. Jiao, G.S. Was, J. Nucl. Mater. 382 (2008) 203-209.

[4] T. Shoji, S. Suzuki, K.S. Raja, J. Nucl. Mater. 258 (1998) 241-251.

[5] I. Karius, W. Gust, Fundamentals of grain and interphase boundary diffusion, Ziegler Press,

Stuttgart, 1988.

[6] H. Kokawa , T. Watanabe, S. Karashima, Philos. Mag. A 44 (1981) 1239-1254.

[7] J.Le. Coze, M. Biscondi, J. Levy, C. Goux, Mem Scient Revue Metall. 70 (1973) 397-407.

[8] J.Le. Coze, M. Biscondi, Can. Metall. Q. 13 (1974) 59-63.

[9] H. Kokawa, M. Shimada, Y.S. Sato, JOM 52 (2000) 34-37.

[10] M. Froment, J. Phys. 36 (1975) c4-c371.

[11] X.R. Qian, Y.T. Chou, Philos. Mag. 45 (1982) 1075-1079.

[12] P.H. Pumphrey, in: G.A. Chadwick and D.A. Smith (Eds), Grain Boundary Structure and Properties, Academic Press, London, 1976, pp.139-200.

[13] T. Watanabe, Res Mechanica 11 (1984) 47-84.

[14] C. Cheung, U. Erb, G. Palumbo, Mater. Sci. Eng. A 185 (1994) 39-43.

[15] E.M. Lehockey, G. Palumbo, Mater. Sci. Eng. A 237 (1997) 168-172. 
[16] G. Palumbo, E.M. Lehockey, P.Lin, JOM. 50 (1998) 40-43.

[17] E.M. Lehockey, G. Palumbo, P. Lin, Scripta Mater. 36 (1997) 1211-1218.

[18] G. Palumbo, U.Erb, MRS. Bul. 24 (1999) 27-32.

[19] S. Spigarelli, M. Cabibbo, E. Evangelista, G. Palumbo, Mater. Sci. Eng. A. 352 (2003) 93-99.

[20] M. Michiuchi, H. Kokawa, Z.J. Wang, Y.S. Sato, K. Sakai, Acta Mater. 54 (2006) 5179-5184.

[21] T.A Kenfield, W.K Appleby, H.J. Busboom, J. Nucl. Mater. 75 (1978) 85-97.

[22] M.J. Makin, G.P. Walters, A.J.E. Foreman, J. Nucl. Mater. 95 (1980) 155-170.

[23] D.G. Brandon, Acta Metall. 14 (1966) 1479-1484.

[24] H. Kokawa, T. Watanabe, S. Karashima, J. Mater. Sci. 18 (1983) 1183-1194.

[25] H. Kokawa, T. Watanabe, S. Karashima, Scripta Metall. 17 (1983) 1155-1159.

[26] K. Uematsu, T. Kodama, Y. Ishida, K. Suzuki, M. Koyama, in: M.K. Bleiberg, J.W. Bennett

(Eds) Radiation Effects in Breeder Reactor Structural Materials, AIME, New York, 1977, pp. 571-589.

\section{Figure Captions}

Fig. 1 Grain boundary character distribution (GBCD) obtained by SEM-EBSD for (a) PNC316-AS, (b) PNC316-GBEM, and (c) PNC316-GBEM20\%CW. (d), (e), and (f) show the GBCDs of PNC316-AS, PNC316-GBEM, and PNC316-GBEM20\% CW after thermal aging at 973 $\mathrm{K}$ for $100 \mathrm{~h}$, respectively. The black and gray lines indicate random and CSL boundaries $(\Sigma \leq 29)$, respectively. 
Fig. 2 TEM images of (a) PNC316-GBEM, (b) PNC316-GBEM20\% CW and thermal-aged (c) PNC316-GBEM, and (d) PNC316-GBEM20\%CW. The aging condition was at 973K for $100 \mathrm{~h}$. The magnified TEM image of PNC316-GBEM20\% CW is shown in (e).

Fig. 3 TEM images of (a) PNC316-AS, (b) PNC316-GBEM, and (c) PNC316-GBEM20\% CW after electron irradiation to $6 \mathrm{dpa}$ at $823 \mathrm{~K}$.

Fig. 4 Dose dependencies of void swelling in PNC316-AS, PNC316-GBEM, and PNC316-GBEM20\%CW during electron irradiation up to 6 dpa at $823 \mathrm{~K}$.

Table 1 Composition of austenitic stainless steel PNC316. (in mass\%) 
Table 1 Composition of austenitic stainless steel PNC316. (in mass\%)

\begin{tabular}{|l|c|c|c|c|c|c|c|c|c|c|c|}
\hline & $\mathrm{C}$ & $\mathrm{Si}$ & $\mathrm{Mn}$ & $\mathrm{P}$ & $\mathrm{Cr}$ & $\mathrm{Ni}$ & $\mathrm{Mo}$ & $\mathrm{B}$ & $\mathrm{Ti}$ & $\mathrm{V}$ & $\mathrm{Nb}+\mathrm{Ta}$ \\
\hline PNC316 & 0.056 & 0.8 & 1.91 & 0.028 & 16.5 & 13.77 & 2.59 & 0.0037 & 0.077 & $<0.01$ & 0.091 \\
& & & & & & & & & \\
(in mass\%)
\end{tabular}

\section{M.SEKINE}

Grain Boundary Engineering of Austenitic Steel PNC316 for use in Nuclear Reactors 

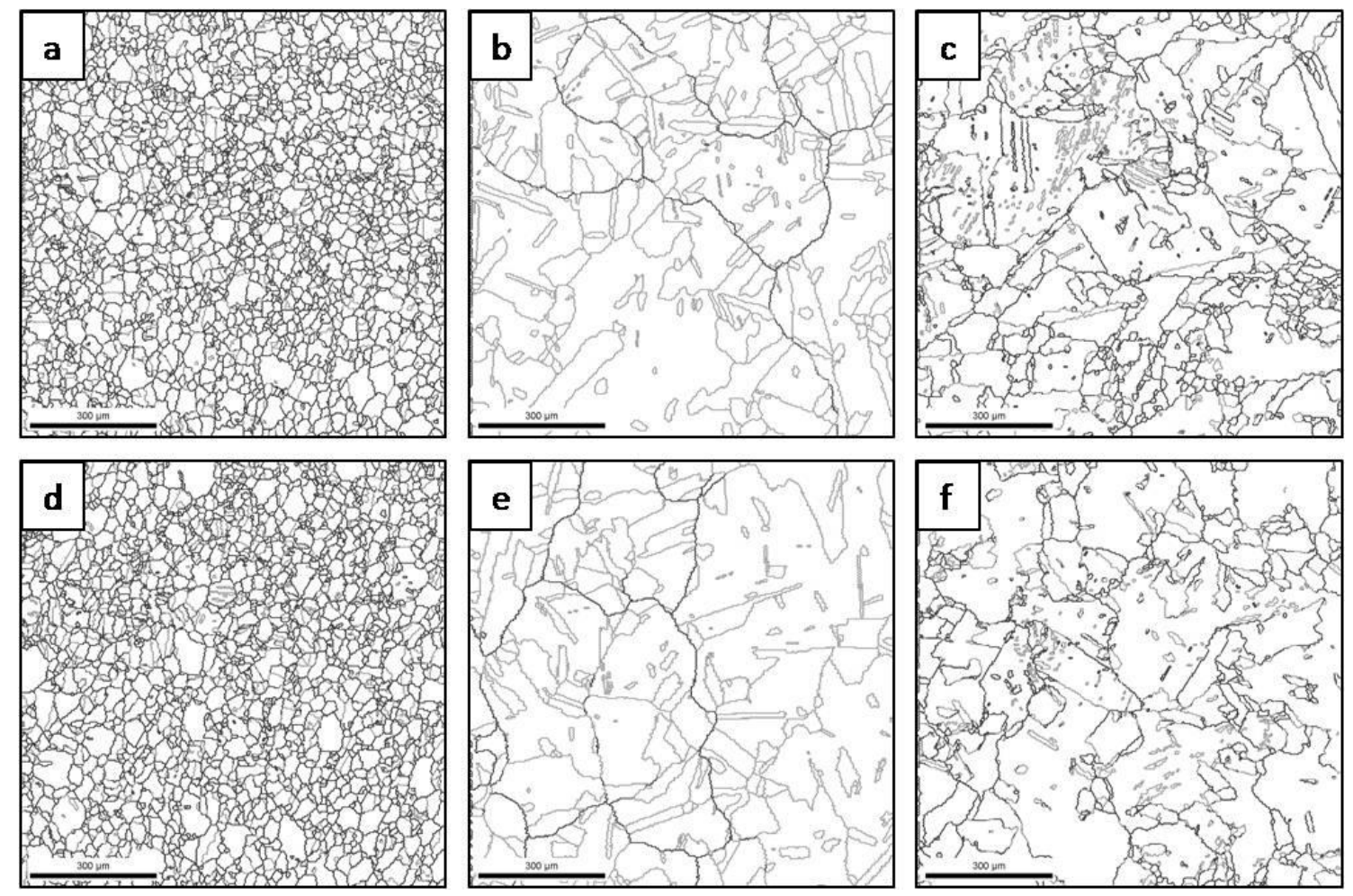

Fig. 1 Grain boundary character distribution (GBCD) obtained by SEM-EBSD for (a)

PNC316-AS, (b) PNC316-GBEM, and (c) PNC316-GBEM20\%CW. (d), (e), and (f) show the

GBCDs of PNC316-AS, PNC316-GBEM, and PNC316-GBEM20\% CW after thermal aging at 973

$\mathrm{K}$ for $100 \mathrm{~h}$, respectively. The black and gray lines indicate random and CSL boundaries $(\Sigma \leq 29)$,

respectively.

\section{M.SEKINE}

Grain Boundary Engineering of Austenitic Steel PNC316 for use in Nuclear Reactors 

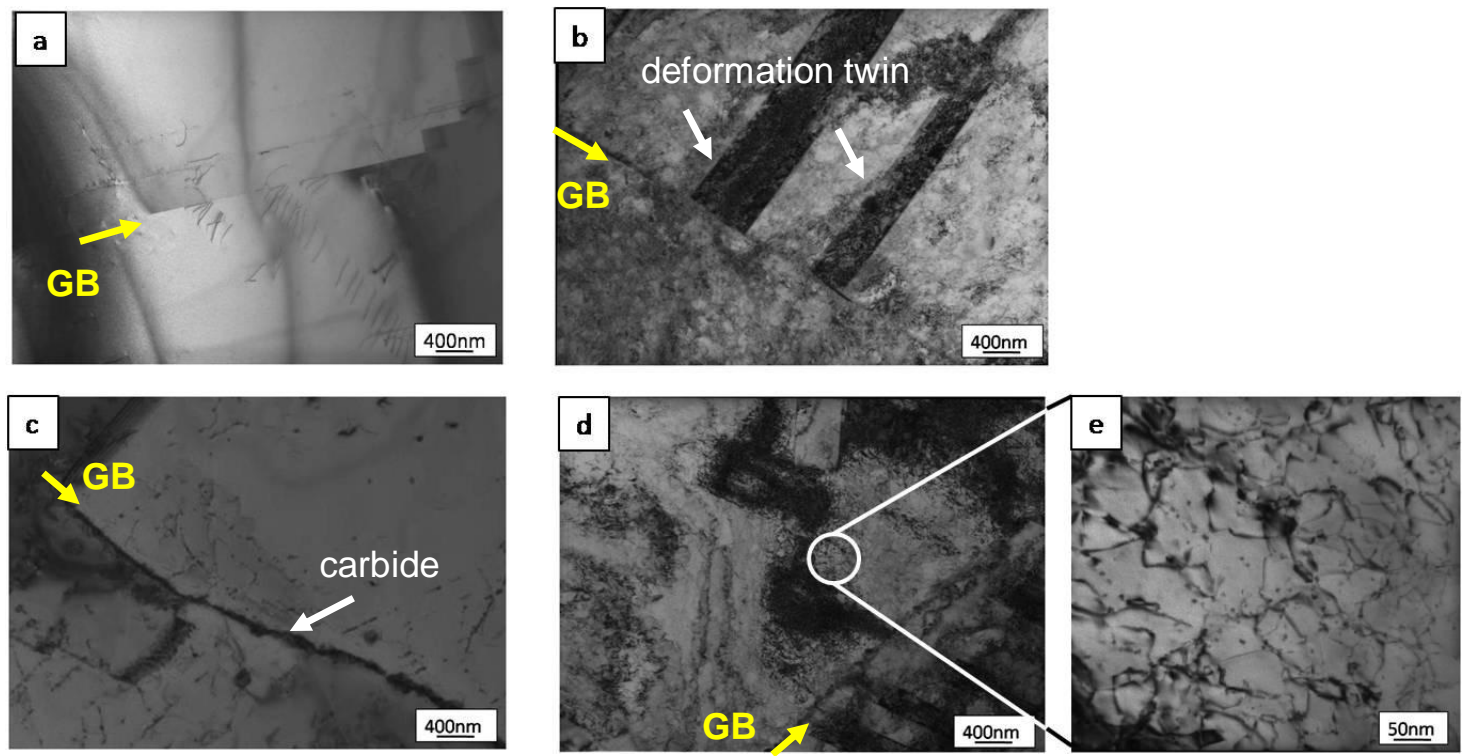

Fig. 2 TEM images of (a) PNC316-GBEM, (b) PNC316-GBEM20\% CW and thermal-aged (c) PNC316-GBEM, and (d) PNC316-GBEM20\%CW. The aging condition was at 973K for $100 \mathrm{~h}$. The magnified TEM image of PNC316-GBEM20\% CW is shown in (e).

M.SEKINE

Grain Boundary Engineering of Austenitic Steel PNC316 for use in Nuclear Reactors 

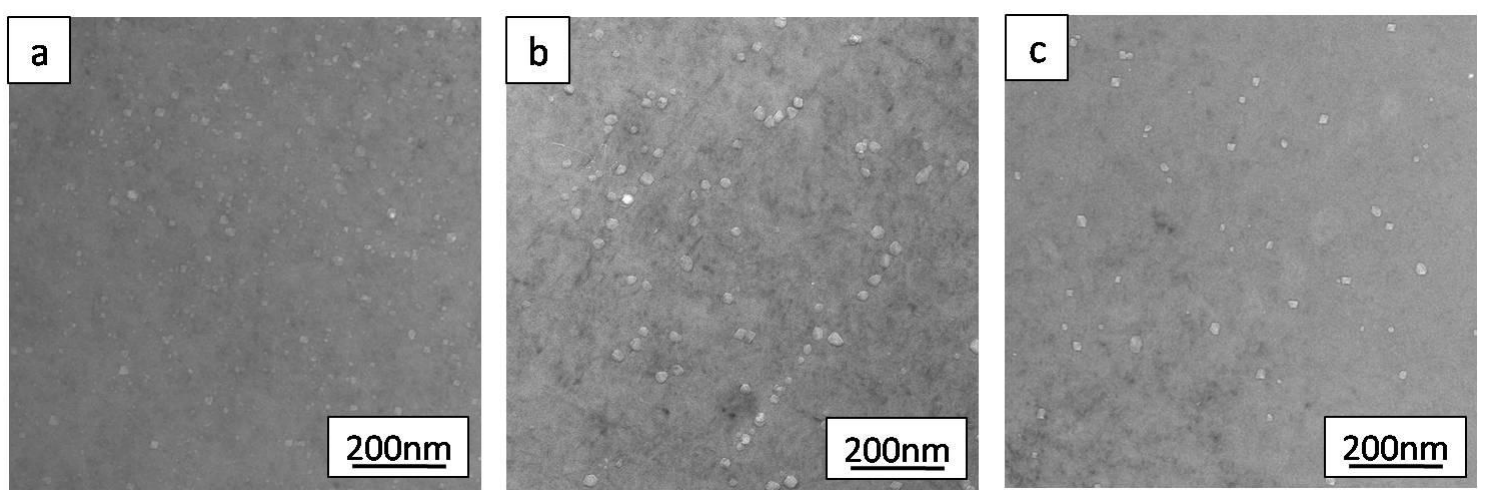

Fig. 3 TEM images of (a) PNC316-AS, (b) PNC316-GBEM, and (c) PNC316-GBEM20\% CW after electron irradiation to $6 \mathrm{dpa}$ at $823 \mathrm{~K}$.

\section{M.SEKINE}

Grain Boundary Engineering of Austenitic Steel PNC316 for use in Nuclear Reactors 


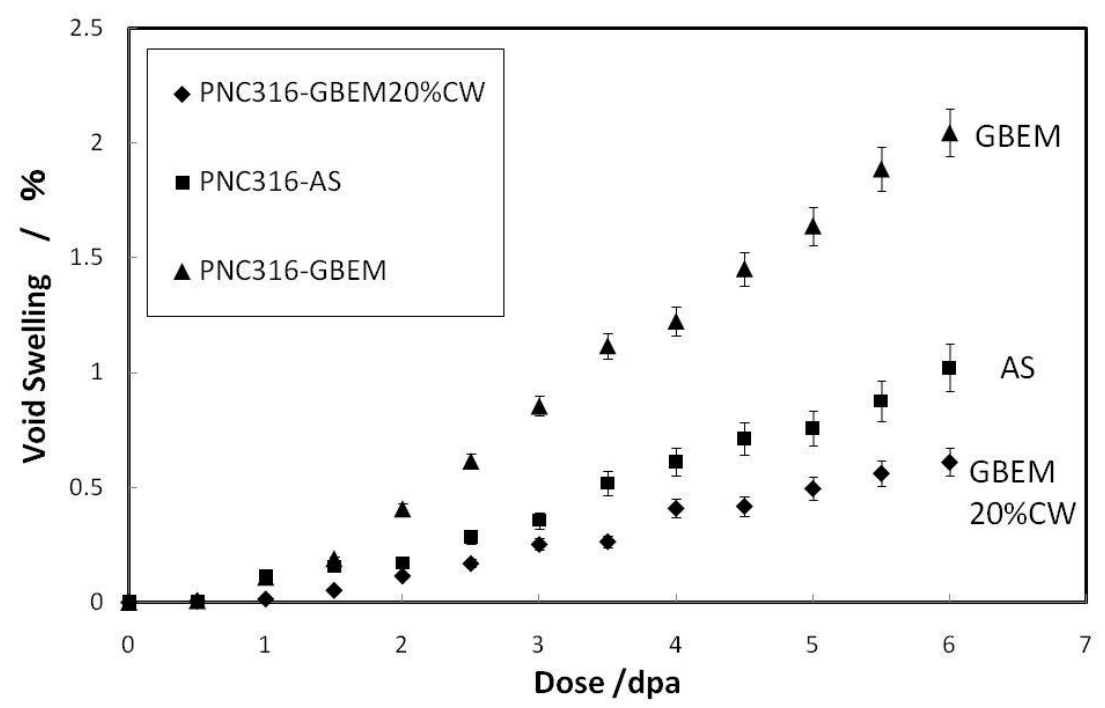

Fig. 4 Dose dependencies of void swelling in PNC316-AS, PNC316-GBEM, and PNC316-GBEM20\%CW during electron irradiation up to 6 dpa at $823 \mathrm{~K}$.

\section{M.SEKINE}

Grain Boundary Engineering of Austenitic Steel PNC316 for use in Nuclear Reactors 